\title{
3D Vector Velocity Estimation using a 2D Phased Array
}

\author{
Pihl, Michael Johannes; Jensen, Jørgen Arendt
}

Published in:

Proceedings of 2011 IEEE International Ultrasonics Symposium

Publication date:

2011

Document Version

Early version, also known as pre-print

Link back to DTU Orbit

Citation (APA):

Pihl, M. J., \& Jensen, J. A. (2011). 3D Vector Velocity Estimation using a 2D Phased Array. In Proceedings of 2011 IEEE International Ultrasonics Symposium (pp. 430-433). IEEE.

http://ewh.ieee.org/conf/ius_2011/index.html

\section{General rights}

Copyright and moral rights for the publications made accessible in the public portal are retained by the authors and/or other copyright owners and it is a condition of accessing publications that users recognise and abide by the legal requirements associated with these rights.

- Users may download and print one copy of any publication from the public portal for the purpose of private study or research.

- You may not further distribute the material or use it for any profit-making activity or commercial gain

- You may freely distribute the URL identifying the publication in the public portal

If you believe that this document breaches copyright please contact us providing details, and we will remove access to the work immediately and investigate your claim. 
Paper presented at the IEEE International Ultrasonics Symposium, Orlando, FL, USA, 2011:

\title{
3D Vector Velocity Estimation using a 2D Phased Array
}

\author{
Michael Johannes Pihl and Jørgen Arendt Jensen
}

Center for Fast Ultrasound Imaging,

Biomedical Engineering group, Department of Electrical Engineering, Bldg. 349, Technical University of Denmark, DK-2800 Kgs. Lyngby, Denmark 


\title{
3D Vector Velocity Estimation using a 2D Phased Array
}

\author{
Michael Johannes Pihl and Jørgen Arendt Jensen \\ Center for Fast Ultrasound Imaging, Department of Electrical Engineering, \\ Technical University of Denmark, DK-2800 Kgs. Lyngby, Denmark
}

\begin{abstract}
A method to estimate the three dimensional (3D) velocity vector is presented is this paper. $3 \mathrm{D}$ velocity vector techniques are needed to measure the full velocity and characterize the complicated flow patterns in the human body. The Transverse Oscillation (TO) method introduces oscillations transverse to the ultrasound beam, which enables the estimation of the transverse velocity. To expand the method from $2 \mathrm{D}$ to $3 \mathrm{D}$, it is proposed to decouple the velocity estimation into separate estimates of $v_{x}, v_{y}$, and $v_{z}$ in combination with a 2D phased matrix array. Through simulations the feasibility of using the TO method for estimation 3D velocity vectors, and the proposed decoupling is

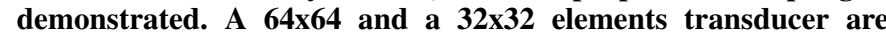
emulated using Field II. Plug flow with a speed of $1 \mathrm{~m} / \mathrm{s}$ in a small region is rotated in the $X Y$-plane. A binary flow example with $\left[v_{x}, v_{y}\right]=[1,0]$ and $[0,1] \mathrm{m} / \mathrm{s}$ shows, that the velocity estimation can be decoupled into the transverse and elevation velocity components. This is substantiated by the results for seven different angles, where the mean and the standard deviation of the estimated speed are $0.97 \pm 0.05 \mathrm{~m} / \mathrm{s}$ and of the angle bias are $-0.73 \pm 3.3^{\circ}$ for the $64 \times 64$ matrix transducer. For the $32 \times 32$ transducer, the mean and standard deviation for the speed are $0.94 \pm 0.11 \mathrm{~m} / \mathrm{s}$ and for the angle bias $-0.48 \pm 7.7^{\circ}$. The simulation study clearly demonstrates, that the new method can be used to estimate the 3D velocity vector using a 2D phased matrix array, and that the velocity vector estimation can be decoupled into separate estimates of $v_{x}, v_{y}$, and $v_{z}$.
\end{abstract}

\section{INTRODUCTION}

Measurements of the blood velocity in the human body play a key role in diagnosing various cardiovascular diseases [1], however, the complicated flow patterns in the circulatory system yet have to be fully characterized. Conventional methods only estimate velocities in one dimension. As the velocities vary as a function of time and space [2], [3], 3D techniques need to be employed to fully estimate and characterize the complicated flow patterns.

Different approaches have been proposed for 2D velocity estimation. A multibeam approach was suggested by Fox [4] where the velocity components are estimated using trigonometry. Speckle tracking (normalized cross-correlation) proposed by Trahey et al. [5] finds the velocity vector by searching for the best match between a kernel and a search region. The directional beamforming approach by Jensen [6] estimates the velocity by cross correlating lines that are beamformed in the direction of the flow.

The Transverse Oscillation method is another technique that solves the angle-dependency problem in traditional ultrasonic flow estimation. The technique was suggested by Jensen and
Munk [7], and in vivo examples were demonstrated in [2]. A similar approach was suggested by Anderson [8].

So far, the technique has only been used for $2 \mathrm{D}$ velocity estimation. Expanding the method from 2D to $3 \mathrm{D}$, it is proposed to estimate $v_{x}, v_{y}$, and $v_{z}$ separately, i.e. to decouple the velocity estimation of $v_{x}$ and $v_{y}$ as well as $v_{z}$. That is contrary to the other methods mentioned above, where the velocity components cannot be decoupled.

This paper will demonstrate the feasibility of using the TO method on simulated data for estimating 3D velocity vectors using a 2D matrix phased array. Furthermore, the purpose is to demonstrate that the velocity estimation can be decoupled into estimating the three velocity components $v_{x}, v_{y}$, and $v_{z}$ separately. A simulation study will serve as a proof of concept. The results are presented in Section IV, followed by a discussion and a conclusion.

\section{The Transverse Oscillation Method}

The basic mechanism, that allows the traditional estimation of axial velocities, is the oscillations in the transmitted ultrasonic pulse. Using the same principle, an introduction of a transverse oscillation in the ultrasound field generates received signals that depend on the transverse motion, and the velocity can be estimated.

\section{A. 3D Velocity Estimation}

For the 2D case, a derivation of the required apodization functions and a description of the respective generated fields for the linear array can be found in [7], [9]. A thorough description and derivation of the velocity estimator used for each decoupled transverse or elevation velocity component is given in [10].

In the $3 \mathrm{D}$ case, it is assumed that the velocity estimates can be decoupled into three independent velocity components: the axial, $v_{z}$, the transverse, $v_{x}$, and the elevation $v_{y}$. The estimated velocity components $v_{x}$ and $v_{y}$ are orthonormal to the scan line. In the setup as illustrated in Fig. 1, the scan line and the $z$-axis coincides, and the velocity components are $v_{x}$, $v_{y}$, and $v_{z}$.

In terms of beamforming, a center line for axial velocity $v_{z}$, two lines in the $X Z$-plane for the transverse velocity $v_{x}$, and two lines in the $Y Z$-plane for the elevation velocity $v_{y}$ as demonstrated in Fig. 1. As a result, the velocity estimation is decoupled into the three components. The pairwise TO beams are steered at specific fixed angles, such that the spacing is a 


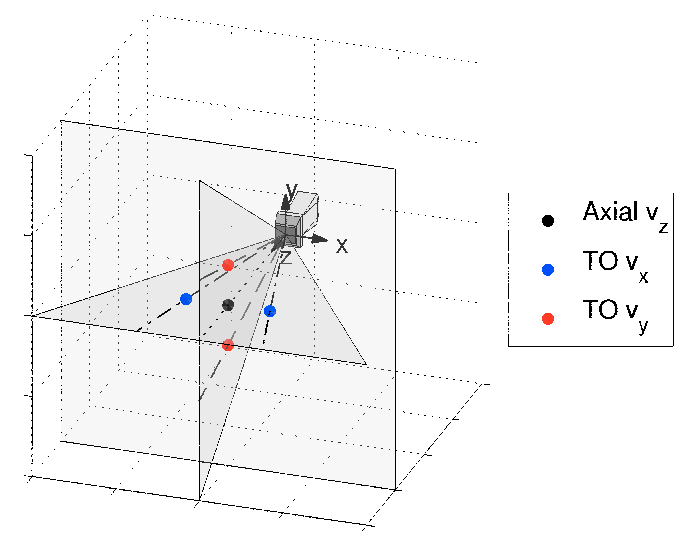

Fig. 1. Beamforming approach for 3D velocity estimation using a 2D matrix phased array transducer. The coordinate system of the transducer is $(x, y, z)$. The dotted line indicates the scan angle, here coinciding with the $z$-axis. Spheres indicate beamformed samples at a given depth: A center line is beamformed for conventional axial velocity $v_{z}$ estimation (black), and two pairwise TO lines are beamformed for estimation of the transverse and elevation velocities $v_{x}$ and $v_{y}$ (blue and red, respectively). The steering angles have been exaggerated for illustration purposes; for the $64 \times 64$ transducer, they are $0.6^{\circ}$ with respect to the $z$-axis. The gray shaded areas illustrate the $X Y$-, $X Z$-, and $Y Z$-planes.

quarter spatial wavelength at every sample depth, because the lateral wavelength increases with depth.

The axial velocity can also be estimated by use of the conventional autocorrelation approach [11]. For the transverse velocity $v_{x}$ the TO method is employed as done previously [12] in $2 \mathrm{D}$ although the apodizations are adapted to the $2 \mathrm{D}$ phased array. As the transverse and elevation velocity components can be decoupled, the field generation, the beamforming approach, and the velocity estimation are identical for $v_{x}$ and $v_{y}$, and follow [12], and is therefore omitted here.

The novelty is that the velocity component in the elevation direction, $v_{y}$, can be measured when using a 2D phased array. Essentially, the process of estimating $v_{y}$ is the same for $v_{x}$. The only difference is that the apodization profiles are rotated 90 degrees.

All five lines are beamformed in parallel in receive based on the same transmission, so only five beamformers in receive are required. However, the method may be expanded to beamform several flow lines in parallel. As the lines are beamformed in parallel, the three velocity components are estimated simultaneously.

\section{Simulations Setup}

The simulations are performed using the ultrasound simulation program Field II [13], [14]. The sampling frequency is $120 \mathrm{MHz}$, and the speed of sound is set to $1480 \mathrm{~m} / \mathrm{s}$.

A 64x64 elements 2D phased array is simulated, however, only 1024 active channels are used. The center frequency is $3 \mathrm{MHz}$, the pitch $\lambda / 2 \mathrm{~mm}$ and the kerf $\lambda / 100 \mathrm{~mm}$. The transducer impulse response is simulated as a 3 cycle pulse at the center frequency multiplied with a Hanning window. A second transducer is simulated with $32 \times 32$ elements, but otherwise with the same characteristics.

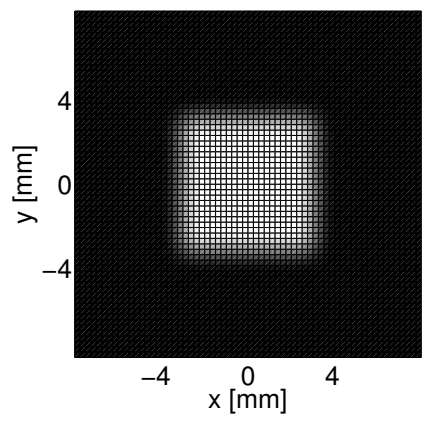

(a) $64 \times 64$ transmit

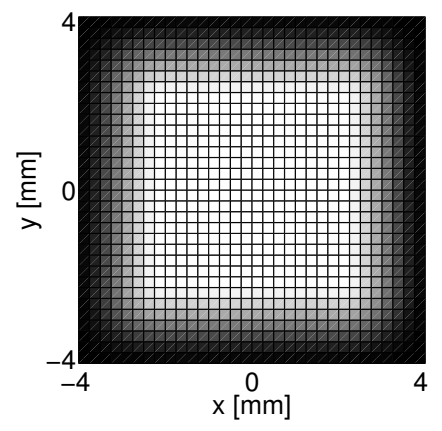

(c) $32 \times 32$ transmit

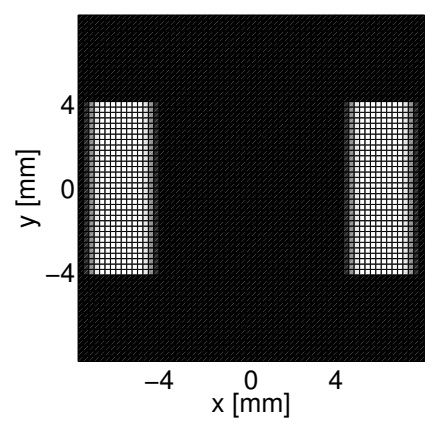

(b) $64 \times 64$ receive

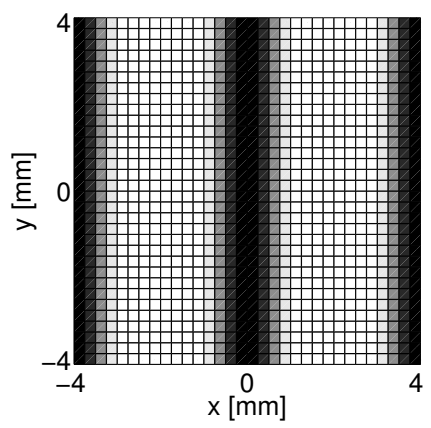

(d) $32 \times 32$ receive
Fig. 2. Apodization functions in transmit and receive for the $64 \times 64$ and $32 \times 32$ apertures. Colorbar: Black $\rightarrow$ white $=0 \rightarrow 1$. Each small square represents an element. For all four apertures, 1024 active channels are used. The transmit apodizations are the same for each receive line, whereas the depicted receive apertures are for the estimation of $v_{x}$.

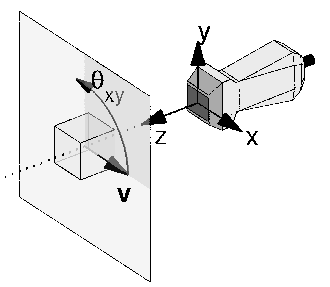

Fig. 3. Illustration of the simulation setup for the 2D transducer. The dotted line is the scan line (here coinciding with the $z$-axis). The light gray shaded plane is orthonormal to the scan line. The cube represents the flow phantom and the black arrow the velocity vector $\mathbf{v}$. In the simulations, the cube is rotated around the $z$-axis at different angles, $\theta_{x y}$, within the darker shaded quadrant.

In transmit, only the center $32 \times 32$ elements of the $64 \times 64$ transducer is used, i.e. 1024 active channels. The focal point is set to $25 \mathrm{~mm}$, whereas the point of interest is at $15 \mathrm{~mm}$. For the $32 \times 32$ transducer, the transmit apodization is identical to the center $32 \times 32$ elements of the $64 \times 64$ transducer, whereas the focus point is moved to $100 \mathrm{~mm}$ to increase the beam width at $15 \mathrm{~mm}$. The transmit apodizations are illustrated in Fig. 2a and 2c. The transmitted pulse is a 8 cycle sinusoid at the center frequency windowed with a Tukey window with a ratio of taper of 0.75 .

For receive, also only 1024 active channels are used. To increase the spatial transverse wavelength, the apodization peaks are placed as far as part as possible. This gives the transducer apodizations as demonstrated in Figs. $2 \mathrm{~b}$ and $2 \mathrm{~d}$. 


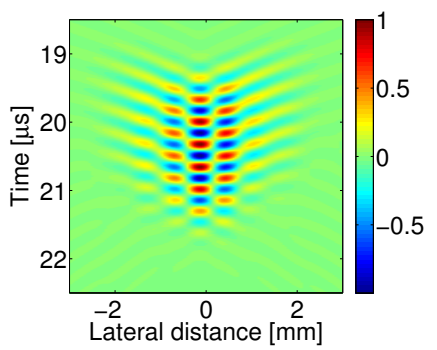

(a)

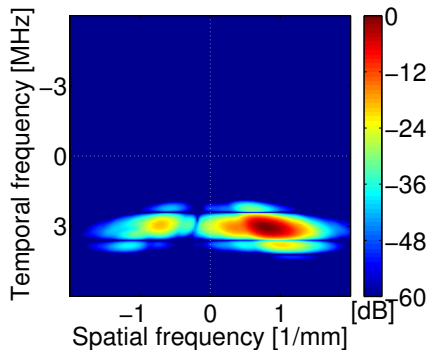

(b)
Fig. 4. (a) Pulse-echo field in normalized pressure for the left TO beam $\left(\mathrm{PEF}_{\text {left }}\right)$ in both the $X Z$ - and $Y Z$-plane. (b) The amplitude spectrum of the 2D spatio-temporal Fourier transform of $\mathrm{PEF}_{\text {left }}+j \cdot \mathrm{PEF}_{\text {right }}$, where both PEFs are complex signals obtained using the Hilbert transform.

The receive apodizations are for the two lines in the $X Z$-plane used for estimating $v_{x}$.

Flow motion is simulated in a $10 \times 10 \times 10 \mathrm{~mm}^{3}$ cube centered at $[x, y, z]=[0,0,15] \mathrm{mm}$ with plug flow as illustrated in Fig. 3 . 10000 scatterers with a Gaussian scattering amplitude distribution with zero mean value and unit variance are randomly, uniformly distributed in the volume. The simulations are performed with a constant speed of $1 \mathrm{~m} / \mathrm{s}$ and a varying angle, $\theta_{x y}$, for the vector flow. The plug flow is rotated around the $z$-axis (i.e. rotations in the $X Y$-plane) as shown in Fig. 3.

\section{RESUlts}

\section{A. The Pulse-Echo Field and its Fourier Domain}

To investigate how well defined the transverse oscillations are, the pulse-echo fields (PEFs) are calculated with Field II, and the 2D Fourier domain is investigated. The obtained pulse-echo field for the $64 \times 64$ transducer with the specified apodizations is illustrated in Fig. 4.

The 2D temporal and spatial Fourier domain is calculated based on the imaginary field obtained when the left beam is the real part, and the right is the imaginary part. The obtained amplitude spectrum is shown in Fig. 4b. The theoretical lateral spatial frequency is $0.85 \mathrm{~mm}^{-1}$, whereas the mean frequency is $0.80 \mathrm{~mm}^{-1}$. This gives a bias of $-6 \%$ between the theoretical and the mean of the simulated spatial frequencies. For the $32 \times 32$ transducer, the values are 3.4 and $4.2 \mathrm{~mm}^{-1}$ for the mean and theoretical spatial frequencies, respectively. The bias is $-20 \%$.

To investigate how well the TO method performs as spatial IQ, the energy should mainly be concentrated in the 4th quadrant. In Fig.4b there is no energy in the 1st and 2nd quadrant, indicating that the Hilbert transform works for all frequencies. For the lateral spatial frequencies, there is an energy leak into the 3rd quadrant, and it is calculated as $E_{l}=E_{3 q} / E_{\text {total }}$, where $E_{3 q}$ is the energy in the 3rd quadrant and $E_{\text {total }}$ is the total energy. In this case, $E_{l}$ is $1.1 \%$ for the $64 \times 64$ transducer and $5.8 \%$ for the $32 \times 32$ transducer. The coefficient of variation is calculated as $C O V=\bar{f}_{x} / D\left(f_{x}\right)$, where $\bar{f}_{x}$ is the estimated mean lateral frequency and $D$ the dispersion of $f_{x}$. COV is $13.4 \%$ and $40.3 \%$ for the $64 \times 64$ and $32 \times 32$ transducers, respectively. These numbers indicate

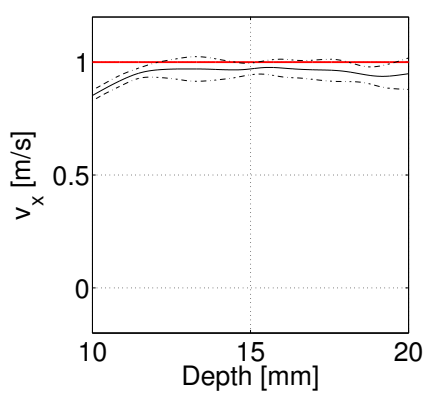

(a) $\theta_{x y}=0$ degrees

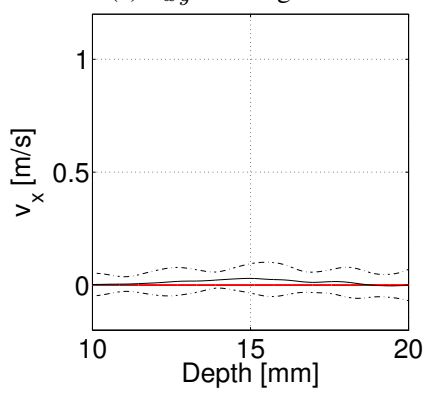

(c) $\theta_{x y}=90$ degrees

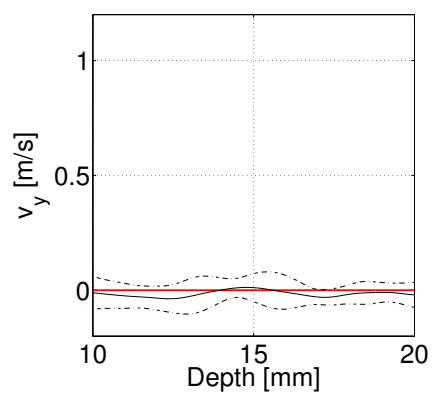

(b) $\theta_{x y}=0$ degrees

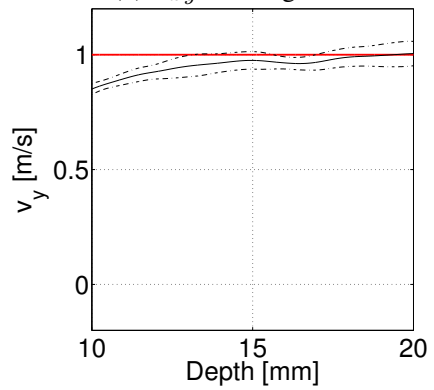

(d) $\theta_{x y}=90$ degrees
Fig. 5. Velocity estimates of $v_{x}$ and $v_{y}$ as a function of $\theta_{x y}$. Thick red line indicates simulated velocity, thin black line the mean of 10 profiles and dot-dashed lines the standard deviation.

that the spatial distribution of frequencies is more biased, and less well defined for the $32 \times 32$ transducer compared to the $64 \times 64$. Therefore, it is expected that the velocity estimates for the $32 \times 32$ transducer will be poorer than for the $64 \times 64$.

\section{B. Velocity Estimates}

Before the velocity estimation, a matched filtration is performed using the time reversed transmitted pulse. No stationary echo cancelling is performed. To obtain temporal IQ data, the Hilbert transform is used to create the analytical signal. $\mathrm{RF}$ averaging is performed on the autocorrelation estimates as suggested in [15]. The velocities are estimated based on 32 shots, and for each flow angle the simulations are repeated 10 times. No bias compensation is performed in the velocity estimations.

Only velocities in the $X Y$-plane are simulated as illustrated in Fig. 3, hence, the results from the estimation of the axial velocity is omitted.

When the simulated velocity is "binary", i.e. $\left[v_{x}, v_{y}\right]=[1,0]$ $\mathrm{m} / \mathrm{s}$ or $[0,1] \mathrm{m} / \mathrm{s}$ (corresponding to $\theta_{x y}=0$ or $90^{\circ}$, respectively), the estimated velocities for the $64 \times 64$ transducer are shown in Fig. 5. As the figure demonstrates, the estimated velocities closely follow the expected value for both angles.

The flow angle is varied from 0 to 90 degrees in steps of 15 degrees in the $X Y$-plane. The velocity components $v_{x}$ and $v_{y}$ at depth $15 \mathrm{~mm}$ are estimated for each angle, and the mean and the standard deviation of the transverse velocity components are calculated. The results for the seven different angles are shown in Fig. 6 for the $64 \times 64$ and the 32x32 transducer, respectively.

The average performance for all 70 estimates at $15 \mathrm{~mm}$ 


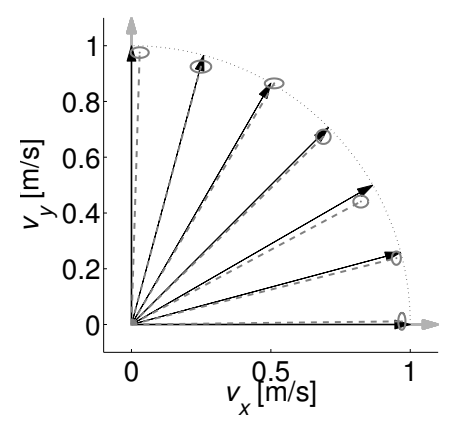

(a) $64 \times 64$

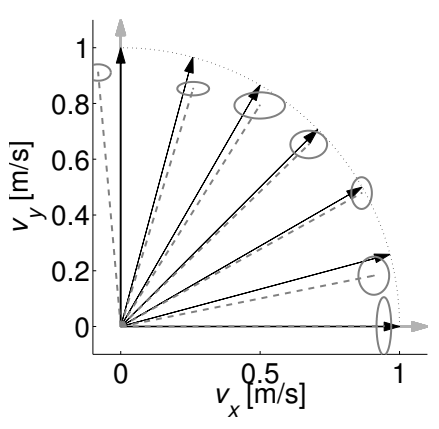

(b) $32 \times 32$
Fig. 6. Velocity estimates as a function of flow angle $\theta$ for the two transducers, respectively. Black arrows (solid) indicate simulated velocities, gray vectors (dashed) the estimated velocities, and gray ellipses (solid) the standard deviations for $v_{x}$ and $v_{y}$, respectively.

TABLE I

Overall PERFormance of THE Estimator

\begin{tabular}{lcc}
\hline Statistics & $64 \times 64$ & $32 \times 32$ \\
\hline Mean speed $[\mathrm{m} / \mathrm{s}]$ & $0.97 \pm 0.047$ & $0.94 \pm 0.11$ \\
Mean angle bias $\left[^{\circ}\right]$ & $-0.73 \pm 3.3$ & $-0.48 \pm 7.7$ \\
\hline
\end{tabular}

depth is shown in Table I for the two transducers. The estimated mean and standard deviation of all speeds and of all biases between the estimated and simulated angle, where the flow angle can be estimated by $\hat{\theta}=\arctan \left(v_{y} / v_{x}\right)$, are listed.

\section{DISCUSSION}

Fig. 5 with its almost "binary" velocity estimates demonstrates, that it is possible to decouple the transverse velocity estimations into $v_{x}$ and $v_{y}$ and estimate them separately. Fig. 6 substantiates this for a number of flow angles. Fig. 6 also shows that the velocity estimates are more uncertain and biased for the $32 \times 32$ than for the $64 \times 64$ transducer, as expected from the PEF and the amplitude spectrum of the 2D spatio-temporal Fourier domain mentioned in the last paragraph of Section IV-A. This is due to the smaller distance between the aperture peaks for the $32 \times 32$ transducer. These findings are more quantitatively illustrated in Table I, where the biases and the standard deviations are higher for the $32 \times 32$ compared to the $64 \times 64$ array. A better match between the theoretical and mean simulated wavelength, will decrease the bias. Optimization of the PEFs is also exptected to reduce the biases and standard deviations.

On average, the standard deviations for the transverse velocities are about $5 \%$ and $10 \%$ for the $64 \times 64$ and the $32 \times 32$ array, respectively, compared to a standard deviation around $1 \%$ for the axial velocity component (not shown). That, however, should be seen in the light of that the conventional axial estimator would wrongfully estimate the speed to be 0 $\mathrm{m} / \mathrm{s}$, when it actually is $1 \mathrm{~m} / \mathrm{s}$ as correctly estimated with the proposed 3D TO method.

The simulations demonstrate that the new method can be used to estimate the $3 \mathrm{D}$ velocity vector when using a $2 \mathrm{D}$ phased array. The beamforming is within the capabilities of modern 3D scanners and the estimators for the three velocity components are on the order of the conventional autocorrelation approach with RF averaging making it suitable for commercial implementations. With clever channel multiplexing the number of active channels needed can also be reduced.

\section{CONCLUSION}

The simulation study demonstrates, that the TO method can be used to estimate the $3 \mathrm{D}$ velocity vector within $5 \%$. The requirements are a 2D phased array, five parallel beamformers, and 1024 active channels. Additionally, the results confirm that the velocity vector estimation can be decoupled into separate and simultaneous estimates of $v_{x}, v_{y}$, and $v_{z}$.

\section{ACKNOWLEDGMENTS}

This work was supported by grant 024-2008-3 from the Danish Advanced Technology Foundation and BK Medical Aps, Denmark.

\section{REFERENCES}

[1] D. H. Evans, J. A. Jensen, and M. B. Nielsen, "Ultrasonic colour Doppler imaging," Interface Focus, vol. 1, no. 4, pp. 490-502, August 2011.

[2] J. Udesen, M. B. Nielsen, K. R. Nielsen, and J. A. Jensen, "Examples of in-vivo blood vector velocity estimation," Ultrasound Med. Biol., vol. 33, pp. 541-548, 2007.

[3] K. L. Hansen, J. Udesen, F. Gran, J. A. Jensen, and M. B. Nielsen, "In-vivo examples of complex flow patterns with a fast vector velocity method," Ultraschall in Med, vol. 30, pp. 471-476, 2009.

[4] M. D. Fox, "Multiple crossed-beam ultrasound Doppler velocimetry," IEEE Trans. Son. Ultrason., vol. SU-25, pp. 281-286, 1978.

[5] G. E. Trahey, J. W. Allison, and O. T. von Ramm, "Angle independent ultrasonic detection of blood flow," IEEE Trans. Biomed. Eng., vol. BME-34, pp. 965-967, 1987.

[6] J. A. Jensen, "Directional velocity estimation using focusing along the flow direction: I: Theory and simulation," IEEE Trans. Ultrason. Ferroelec., Freq. Contr., vol. 50, pp. 857-872, 2003.

[7] J. A. Jensen and P. Munk, "A New Method for Estimation of Velocity Vectors," IEEE Trans. Ultrason., Ferroelec., Freq. Contr., vol. 45, pp. 837-851, 1998.

[8] M. E. Anderson, "Multi-dimensional velocity estimation with ultrasound using spatial quadrature," IEEE Trans. Ultrason., Ferroelec., Freq. Contr., vol. 45, pp. 852-861, 1998.

[9] J. Udesen and J. A. Jensen, "Investigation of Transverse Oscillation Method," IEEE Trans. Ultrason., Ferroelec., Freq. Contr., vol. 53, pp. 959-971, 2006.

[10] J. A. Jensen, "A New Estimator for Vector Velocity Estimation," IEEE Trans. Ultrason., Ferroelec., Freq. Contr., vol. 48, no. 4, pp. 886-894, 2001.

[11] C. Kasai, K. Namekawa, A. Koyano, and R. Omoto, "Real-Time TwoDimensional Blood Flow Imaging using an Autocorrelation Technique," IEEE Trans. Son. Ultrason., vol. 32, pp. 458-463, 1985.

[12] M. J. Pihl and J. A. Jensen, "Transverse oscillations for phased array vector velocity imaging," in Proc. IEEE Ultrason. Symp., 2010, pp. $1323-27$.

[13] J. A. Jensen, "Field: A Program for Simulating Ultrasound Systems," Med. Biol. Eng. Comp., vol. 10th Nordic-Baltic Conference on Biomedical Imaging, Vol. 4, Supplement 1, Part 1, pp. 351-353, 1996.

[14] J. A. Jensen and N. B. Svendsen, "Calculation of Pressure Fields from Arbitrarily Shaped, Apodized, and Excited Ultrasound Transducers," IEEE Trans. Ultrason., Ferroelec., Freq. Contr., vol. 39, pp. 262-267, 1992.

[15] T. Loupas, J. T. Powers, and R. W. Gill, "An axial velocity estimator for ultrasound blood flow imaging, based on a full evaluation of the Doppler equation by means of a two-dimensional autocorrelation approach," IEEE Trans. Ultrason., Ferroelec., Freq. Contr., vol. 42, pp. 672-688, 1995. 